Rev. Biol. Trop., 48(2/3): 361-364, 2000

www.ucr.ac.cr www.ots.ac.cr www.ots.duke.edu

\title{
Crustáceos asociados a macroalgas en Bajo Pepito, Isla Mujeres, Caribe mexicano
}

\author{
Concepción Campos Vázquez \\ Depto. Ecología Acuática, ECOSUR, Apdo. Postal 424, Chetumal, Quintana Roo. 77000 México. Fax (21666) ext. \\ (240); ccampos@ecosur-qroo.mx
}

Recibido 13-V-1999. Corregido 26-XI-1999. Aceptado 13-XII-1999.

\begin{abstract}
Crustaceans associated with macroalgae were collected for one year by scuba diving in Bajo Pepito, Isla Mujeres, mexican Caribbean. A total of 148 organisms were found: three orders, 11 families, 18 genera and 19 species in nine types of associations. The order with highest abundance was Isopoda (112), followed by Amphipoda (20) and Decapoda (16).
\end{abstract}

Key words: Crustacea, macroalgaes, taxonomy, Isla Mujeres.

La abundancia de la epifauna sobre pastos y macroalgas bénticos ha sido notada desde principios de este siglo (Mukai 1971).

En ciertas áreas costeras la densa vegetación de algas y praderas de fanerógamas marinas juegan un papel fundamental en la producción primaria, ya que la mayoría de los animales dependen para su sobrevivencia directa $o$ indirectamente de este grupo de plantas para su alimentación (Ganesan 1983 cit. por Quan-Yong 1999).

Las macroalgas proporcionan además de alimento, protección, zonas de reproducción y crianza para una amplia variedad de organismos marinos (Strombus gigas Linné cit. por Quan-Young 1999).

Ha sido bien reconocido que los herbívoros marinos son participantes activos en las interacciones planta-herbívoro y pueden influir grandemente en la estructura de las comunidades algales bénticas (Cronin \& Hay 1966).

Los mesoherbívoros (incluidos aquí pequeños cangrejos, camarones, anfípodos e isópodos) juegan un papel importante en algunas co- munidades marinas, hay en efecto evidencias de que los mesoherbívoros ayudan en un principio a la dispersión de esporas y facilitación de la sucesión y por último a la longevidad de macrophytas e invertebrados sésiles, además mantienen a otros organismos como peces y aves acuáticas bien alimentados (Brawley 1992).

Este estudio proporciona un listado de las especies de crustáceos encontradas en $\mathrm{Ba}$ jo Pepito, y menciona sus asociaciones con macroalgas.

\section{MATERIALES Y MÉTODOS}

Se realizaron recolectas generales mensuales de macroalgas entre febrero de 1997 y enero de 1998 en un manto mixto de Gracilaria crassissima y Gracilaria cornea el cual ocupa un área de aproximadamente $2 \mathrm{Km}^{2}$ localizándose al oeste de la parte sur de Isla Mujeres, en una zona llamada Bajo Pepito $\left(21^{\circ} 12^{\prime} 21^{\prime \prime} \mathrm{N}, 86^{\circ}\right.$ $44^{\prime} 40^{\prime \prime}$ ); es una zona de aguas claras y corrientes fuertes todo el año, con una profundidad 


\section{CUADRO 1}

Especies de crustáceos recolectadas en Bajo Pepito, Isla Mujeres. Se indica la abundacia total, número de ovígeras, hembras y machos por especie y las algas con las cuales se asocian.

Crustáceos

Periclimenes americanus (Kingsley, 1878)

Clibanarius tricolor (Gibbes, 1850)

Dromidia antillensis Stimpson, 1858

Epialtus dilatatus A. Milne Edwards, 1878

Macrocoeloma subparallelum (Stimpson,1860)

Microphrys antillensis Rathbun, 1920

Microphrys bicornutus (Latreille, 1825)

Mithrax forceps (A. Milne Edwards, 1875)

Tyche emarginata White, 1847

Platyactaea setigera (H. Milne Edwards, 1834)

Stenetrium stebbingi Richardson, 1902

Anopsilana jonesi Kensley, 1987

Cirolana parva Hansen, 1890

Paracerceis caudata (Say, 1818)

Cymodoce ruetzlery Kensley, 1984

Erichsonella filiformis (Say,1818)

Leucothoe spinicarpa (Abildgaard, 1798)

Elasmopus rapax Costa, 1853

Maera inaequipes (A. Costa 1857)

\begin{tabular}{|c|c|c|c|c|}
\hline $\mathrm{Ab}$ & $\mathrm{O}$ & $\mathrm{H}$ & $\mathbf{M}$ & $\mathrm{Ma}$ \\
\hline 3 & 1 & 2 & & Pd, Uf, Csp, B \\
\hline 3 & 1 & 1 & 1 & Csp \\
\hline 1 & & 1 & & Var \\
\hline 1 & 1 & & & $\mathrm{Pd}, \mathrm{Uf}$ \\
\hline 1 & & & 1 & Sv \\
\hline & 1 & & 1 & Sv \\
\hline 3 & 1 & 1 & 1 & Lv \\
\hline 1 & & 1 & & $\mathrm{Hi}, \mathrm{B}$ \\
\hline 1 & 1 & & & B \\
\hline 1 & & 1 & & Csp, Var \\
\hline 5 & & 5 & & $\mathrm{Pd}, \mathrm{B}$ \\
\hline 1 & & 1 & & Uf \\
\hline 10 & & 6 & 4 & Pd, Csp, B \\
\hline 90 & & 70 & 20 & Pd, Csp, Hi, Sz, B \\
\hline 4 & & 4 & & Pd, Csp, Var, B \\
\hline 2 & & 2 & & Csp, B \\
\hline 1 & & 1 & & Var \\
\hline 4 & & 3 & 1 & Pd, Uf, B \\
\hline 15 & & 9 & 6 & Csp, Var \\
\hline
\end{tabular}

$\mathrm{Ab}=$ Abundancia; $\mathrm{O}=$ Ovígera; $\mathrm{H}=$ Hembra; $\mathrm{M}=$ Macho; $\mathrm{Ma}=$ Macroalgas; $\mathrm{Pd}=$ Penicillus dumetosus $; \mathrm{Uf}=$ Udotea flabellum; Csp=Caulerpa sp.; Var=Varias macroalgas; $\mathrm{Sv}=$ Sargassum vulgare; $\mathrm{Lv}=$ Lobophora variegata; $\mathrm{H}=$ Halimeda incrassata; $\mathrm{Sz}=$ Stipopodium zonale; $\mathrm{B}=\mathrm{Blanquizal}$.

aproximada entre 3 y 4 metros, con fondo rocoso-arenoso, predominantemente rocoso, con rocas no mayores de $50 \mathrm{~cm}$ de diámetro (GómezPoot 1999). Las macroalgas se recolectaron a mano o con la ayuda de un cuchillo para buzo tratando de tomar una planta completa para cada especie diferente, incluyendo la base o disco de fijación; se guardaron en bolsas de plástico etiquetadas (con datos de lugar y fecha); posteriormente se fijaron en formol con agua marina al 4\% (Quan-Young 1999).

En el laboratorio los crustáceos fueron separados de las macroalgas y preservados en alcohol al 70\%. En la identificación taxonómica se utilizaron las claves de crustáceos decápodos de Abele y Kim (1986), la guía para isópodos marinos del Caribe de Kensley y Schotte (1989) y para anfípodos las claves de Bousfield (1973) para anfípodos gammaridos, así como también el trabajo realizado por OlivaRivera y Jiménez-Cueto (1992) de anfípodos de Sian Ka'an. Todas las especies identificadas se depositaron en la colección de referencia de ECOSUR (QNR.IN.021.0497).

\section{RESULTADOS}

Durante la investigación se registraron un total de 148 organismos agrupados en 3 ordenes, 11 familias, 18 géneros y 19 especies de crustáceos en 9 diferentes tipos de asociaciones. El orden que presentó la mayor abundancia fue Isopoda, seguido de Amphipoda y Decapoda. El número de hembras con relación a los machos fue mayor para la mayoría de los órdenes estudiados. Los organismos de menor talla como los anfípodos e isópodos superan en abundancia a los decápodos, sin embargo estos últimos presentaron una mayor riqueza de especies.

\section{DISCUSIÓN}

Se identificaron un total de 19 especies pertenecientes a tres ordenes Decapoda (10), Isopoda (6) y Amphipoda (3) de las cuales Tyche emarginata, Stenetrium stebbingi, Anopsilana jonesi y Cymodoce ruetzleri fueron nuevos 
informes para Isla Mujeres, lo que representa un $21 \%$ de nuevos registros para la isla, esto podría deberse a pocos trabajos realizados en Isla Mujeres sobre crustáceos y específicamente en Bajo Pepito en donde ésta es la primera investigación en realizarse.

El $89 \%$ del total de los organismos fueron hembras, sin embargo no se puede concluir que las macroalgas constituyan sitios de reproducción, ya que únicamente en el orden Decapoda se encontraron ovígeras.

Las macroalgas constituyen sitios de refugio y alimentación para los crustáceos recolectados. Se han realizado estudios de herbivoría en la zona con cangrejos (Mithrax forceps, $M$. coryphe, Platyactaea setigera) y se observó que éstos se alimentan de forma considerable de las algas rojas (Gracilaria cornea y G. crassissima) (Gómez-Poot 1999), además éstas le sirven de camuflaje para pasar desapercibidos a sus predadores, Microphrys bicornutus tapiza su caparazón con Lobophora variegata, esta alga estuvo presente en todos los meses de muestreo y también fue informada como epífita. (Quan-Young 1999)

En experimentos realizados por Herrnkind \& Butler IV 1986 reportan que la depredación sobre langostas juveniles fue muy elevado en blanquizales, reducida en masas algas y pastos y mucho mas reducida en praderas de algas densas. Lo anterior explica el bajo porcentaje (9\%) de organismos encontrados en zona de blanquizal.

Los mesoherbívoros son pequeños invertebrados forrajeros, comprendidos aquí animales más grandes que un copépodo promedio pero mas pequeños que $2.5 \mathrm{~cm}$. (Brawley 1992), en esta investigación la mayor abundancia se concentró en organismos de talla pequeña como son Isópodos y Anfípodos, lo cual pudo haberse debido a que el método de muestreo no fue eficaz para la recolecta de organismos de tallas mayores como son los decápodos.

\section{AGRADECIMIENTOS}

Agradezco a María de los Angeles Díaz Martín, Lizette Quan Young, Jorge Gómez Poot y Julio Espinosa Avalos por la recolecta y separación de los crustáceos, así como también la identificación taxonómica de las macroalgas incluidas en este trabajo, así como también a Sergio Salazar Vallejo por la revisión del manuscrito.

\section{RESUMEN}

Como resultado de muestreos mensuales durante un año en Bajo Pepito, Isla Mujeres, Caribe mexicano, se presentan los crustáceos asociados a macroalgas recolectadas por buceo autónomo. Se hallaron un total de 148 organismos: tres ordenes, 11 familias, 18 géneros y 19 especies en nueve tipos de asociaciones. El orden con mayor abundacia fue Isopoda (112) seguido de Amphipoda (20) y Decapoda (16).

\section{REFERENCIAS}

Abele, L. G. \& W. Kim 1986. An illustrated guide to the marine decapod crustaceans of Florida. Dept. Environ. Reg. Tech. Ser. 8: 1-760.

Bousfield, E. L. 1973. Shallow-water gammaridean amphipoda of New England. Cornell University, Ithaca, New York. 312 p.

Brawley, S.H. 1992. Mesoherbivores. p 235-263. In: D.M John, S.J. Hawkins \& J.H. Price (eds.) Plant- animal interaction in the marine benthos. Clarendon, Oxford.

Cronin, G. \& M. E. Hay. 1966. Induction of seaweed chemical defenses by amphipod grazing. 77: 2287-2301.

Gómez-Poot, J. M. 1999. Efecto de la herbivoría en Gracilaria crassisima y Gracilaria cornea (Rhodophyta) de la zona sur de Isla Mujeres, Quintana Roo, México. Tesis de Licenciatura. Instituto Tecnológico de Chetumal, Quintana Roo, México. 93p.

Herrnkind, W. F. \& M. J. Buttler IV. 1986. Factors regulating postlarval settlement and juvenile microhabitat use by spiny lobsters Panulirus argus. 34: 23-30.

Kensley, B. \& M. Schotte. 1989. Guide to the Marine Isopod Crustaceans of the Caribbean. Smithsonian Institution, Washington, D.C. 308 p.

Markham, J. C., F. E. Donath-Hernandez, J.L. VillalobosHiriart \& A.C. Díaz-Varriga. 1990. Notes on the shallow-water Marine Crustacea of the Caribbean Coast of Quintana Roo, Mexico. Anales Inst. Biol. Univ. Nac. Autón. México. Ser. Zool. 61: 405-446. 
Mukai, H. 1971. The phytal animals on tha thalli of Sargassum serratifolium in the Sargassum region, with reference to their seasonal fluctuations. Mar. Biol. 8: 170-182.

Oliva-Rivera, J. J. \& M. S. Jiménez-Cueto. 1992. Anfípodos bentónicos (Crustacea: Peracarida) de la Reserva de la Biosfera de Sian Ka'an, Quintana Roo, p. 169195. In: Navarro, D. \& E. Suárez (eds.) Diversidad Biológica en la Reserva de la Biosfera de Sian Ka'an, Quintana Roo, México. CIQRO. Chetumal, Quintana Roo, México..
Quan-Young, L. I. 1999. Macroalgas asociadas a un manto mixto de Gracilaria crassissima y G. cornea (Rodophyta) en Isla Mujeres, Q.Roo. Tesis de Licenciatura, Instituto Tecnológico de Chetumal, Chetumal, Quintana Roo, México. 116 p. 